\title{
Comparative Study on Fuzzy Supervision and Gain Scheduling for Nonlinear Level Control System
}

\author{
Chalidia Nurin Hamdani ${ }^{1, *}$ Wasis Waskito Adi ${ }^{2}$ Dimas Okky Anggriawan ${ }^{3}$ \\ ${ }^{1,2}$ Refinery Instrumentation Engineering Study Program, Politeknik Energi dan Mineral Akamigas, 58315, Indonesia
${ }^{3}$ Electrical Engineering Department, Politeknik Elektronika Negeri Surabaya, 60111, Indonesia
${ }^{*}$ Corresponding author. Email: hamdani113@gmail.com
}

ABSTRACT

Nonlinear system is always become interesting topic to be researched. Level system is the one of them. Deadtime, changeable dynamics, etc make the conventional controller like PID have to be improved and optimized to face this system. In this paper, we propose a comparative study about PID control which is optimized using two different methods. First method is PID-fuzzy supervision: the fuzzy is used to supervise the PID parameters based on desired operation points and performance. Second method is PID-gain scheduling: the optimal parameters is mapped in scheduling algorithm for the PID . Both methods is evaluated and compared in terms of transient response transmitter in some set point conditions by MATLAB simulation. The simulation result shown that the PID-fuzzy supervision have a better performance than the PID-gain scheduling. PID-fuzzy supervision give a faster response with 0.9 second of time constant deviation and 0.7 second of settling time deviation against PID-gain scheduling in operation point of 22 percent. Furthermore, this result is also confirmed by the value of ITAE. In same operation point, PID-fuzzy supervision ITAE $(249,4779)$ is smaller than PID-gain scheduling ITAE $(307,0614)$.

Keywords: Nonlinear System, Fuzzy Supervision, Gain Scheduling.

\section{INTRODUCTION}

In industrial process, known 4 basic process system must be controlled: level, flow, pressure, and temperature system. This paper is focused on one of them, level system. In its application, level system has characteristics of slow response. This is caused by system's deadtime and delay time. Moreover, the dynamic of level system can change based on set point changes. Because of those, level system is classified as nonlinear system [1][2]. This make the level system is very challenging to be researched.

Proportional, Integral and Derivative (PID) controllers are still common in industry. This controller is the most important control system used to control industrial process, due to its simple and easy design, low cost and wide range of application [3]. However, there are still many challenges in PID design itself.

In 1942, Ziegler-Nichols have introduced tuning formula for PID controller based on system timeresponse and their experiences [4][5]. Uncertainty in generating an appropriate parameter is the weakness of this method. In [6], Chen and Seborg have introduce
Direct Synthesis (DS) method can be used for PID controller design based on process model will be controlled. DS methods offer a better formula in controller design so desired closed-loop system response can be obtained. This method can be used for PID controller for linear system, but it still needs optimization formula to face a nonlinear system. The relative difference between the linear and nonlinear controller is presented in [7].

Some researchers were studied on the use of nonlinear systems and special method to improve the PID controller [8][9]. Moreover, some methods were designed using PID structures to take the advantages and improve the performance [10]. The main problem of these method is an unapplicable algorithm. In field, the PID controller algorithm have been design with default program in some hardware product like PLC, DCS etc. A user cannot modify the PID algorithm inside. So, the most efficient and possible thing to do is to create new hardware with new algorithm/method to enhance the PID controller in it.

Studies about control method combined with PID controller is quite a lot. In [11], neural network and fuzzy 
logic was designed together with PID controller for heating system. The result is good: fast response, smallest overshoot and lowest rise and settling time. But, still, the algorithm is too complex to be implemented.

The simpler method was proposed in [12]. Gain scheduling PID control was designed for unmanned surface vessel. This method makes the PID parameters can change with respect to the set point determined. In the same way, but different method of calculation, in [13] fuzzy logic was designed to tune PID parameters based on error and delta-error value. The most important thing in deal with nonlinear system is how to make change of control effects in case of presence of nonlinearity. It makes these topics interesting to be investigated.

In this paper, we investigate the model for level system by a control design that is simple yet effective. We clarify the problem and control design in the next chapter. In Section III, simulation results and discussions of the control design are demonstrated. The summary of the research findings is described in the conclusion section.

\section{METHOD}

Level system we used in this research consists of process tank, reservoir tank, motor pump, control valve and DP transmitter. This system was treated as it deserves, as a nonlinear system. In some application with boundary operation point, the system can be linearized so the conventional PID can handle it. However, in wide operation point (unbounded), the system is nonlinear, single PID parameters cannot handle it. Needed an extra time and energy to tune the PID controller regarding to the nonlinearity.

Table 1. Liquid level system transfer function in some operation points

\begin{tabular}{|c|c|c|}
\hline Operation Point & Transfer Function & Estimation Data Fit \\
\hline \multirow[t]{2}{*}{15} & $2,597 e^{-0,192 s}$ & $81,47 \%$ \\
\hline & $\overline{1+1,807 s}$ & \\
\hline \multirow{2}{*}{20} & $2,390 e^{-0,191 s}$ & \\
\hline & $\overline{1+2,081 s}$ & $90,83 \%$ \\
\hline \multirow[b]{2}{*}{25} & $2,486 e^{-0,187 s}$ & \\
\hline & $\overline{1+2,919 s}$ & $92,16 \%$ \\
\hline \multirow{2}{*}{30} & $1,896 e^{-0,197 s}$ & \\
\hline & $\overline{1+2,341 s}$ & $90,85 \%$ \\
\hline
\end{tabular}

In this paper, we generate mathematical models for level system for some operation points determined using black box method. By using some operation points determined as modelling reference and the help of System Identification Toolbox on MATLAB software, so we can obtain mathematic models of the system as can be seen on Table 1.

The design consists of 2 sub control systems. To make sure the objectives is met, PID parameters is calculated by using direct synthesis method. Calculating is done for all operation point determined along with desired transient response performance. Then, for modifying the PID, gain scheduling and fuzzy supervision is designed using operation point determined and PID parameters obtained.

General equation of PID controller

$G_{c}(s)=K_{p}\left(1+\frac{1}{T_{i} s}+T_{d} s\right)$

With $\mathrm{Kp}, \mathrm{Ti}$ and $\mathrm{Td}$ is proportional gain, integral time, and derivative time of PID respectively. PID designed using Direct Synthesis (DS). This method allow designer to design PID controller based on system model and desired closed loop system model. From closed-loop control system transfer function and $1^{\text {st }}$ order of desired closed loop system model, we can describe the controller equation $G_{c}(s)$ as:

$G_{c}(s)=\frac{\tau}{K\left(\tau_{c}+\theta\right)}\left(1+\frac{1}{\tau s}\right)$

Equation (2) is the controller equation have to be met so the desired characteristics of closed-loop system model can be achieved. By comparing equation (1) and (2) we can obtain:

$K_{p}=\frac{1}{K} \frac{\tau}{\theta+\tau_{c}}$

$T_{i}=\tau$

$T_{d}=0$

From equation (3) - (5), we can see that the level system can be controlled moderately by using Proportional and Integral control. To calculate the PID parameters for 4 operation points, we must determine 4 desired closed-loop system models. Table 2 shows the desired closed-loop system models and PID parameters related to it. 
Table 2. Desired closed-loop system models

\begin{tabular}{ccccc} 
Operation Points & Desired Models & Kp & Ti & Td \\
\hline $\mathbf{1 5}$ & $\frac{e^{-0,192462 s}}{3 s+1}$ & 0,21801684 & 1,8074 & $\mathbf{0}$ \\
$\mathbf{2 0}$ & $\frac{e^{-0,190940 s}}{3 s+1}$ & 0,3974293 & 2,0816 & $\mathbf{0}$ \\
$\mathbf{2 5}$ & $\frac{e^{-0,1870462 s}}{3 s+1}$ & 0,36837389 & 2,91886 & $\mathbf{0}$ \\
$\mathbf{3 0}$ & $\frac{\boldsymbol{e}^{-\mathbf{0 , 1 9 7 3 s}}}{\mathbf{3 s + \mathbf { 1 }}}$ & $\mathbf{0 , 3 8 6 1 5 1 6 8}$ & $\mathbf{2 , 3 4 1 5}$ & $\mathbf{0}$ \\
\hline
\end{tabular}

For gain scheduling control, we used simple premiseconclusion rules for operation points and PID parameters calculated. So, the rules are:

$$
\text { if } 15<=O P<20 \text { then } K p=0,21801684 \text { and } \mathrm{Ti}
$$
$=1,8074$

If $20<=O P<25$ then $K p=0,3974293$ and $T i=$ 2,0816

If $25<=O P<30$ then $\mathrm{Kp}=0,36837389$ and $\mathrm{Ti}=$ 2,91886

If $O P=>30$ then $K p=0,38615168$ and $T i=2,3415$
These rules will generate crisp PID parameters regarding to the set point given with strict border.

The fuzzy supervision was designed using operation points as input and PID parameters calculated as output. We used Mamdani fuzzy inference with one input and two output, AND minimum operation, minimum function on implication, maximum function on aggregation and centroid function on defuzzification. For input, we used triangle function as can see on Figure 1, to mapping the operation points. Two output is proportional gain and integral time whose was mapped using triangle and trapezoidal function as can see on Figure 2.

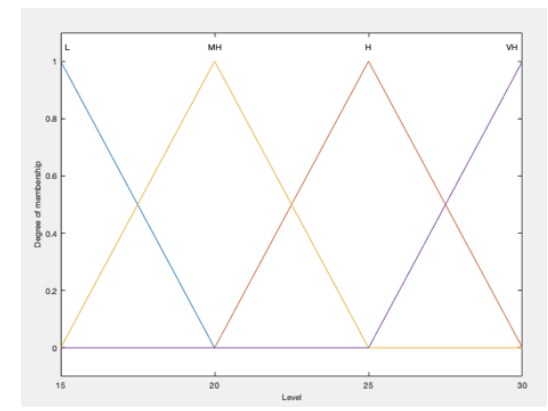

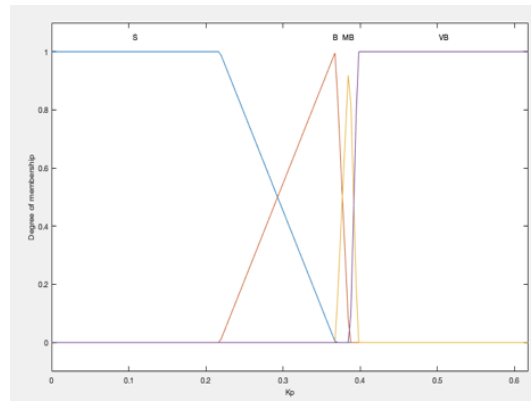

b

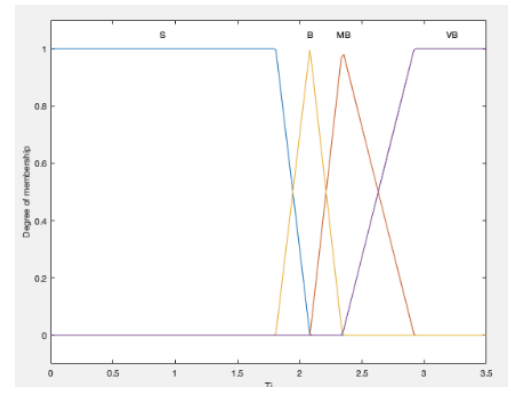

$\mathrm{c}$

Figure 1 (a) Input (b) Kp output and (c) Ti output membership function

The rule based was used in the fuzzy logic is:

If (Operation Point is Low) then ( $k p$ is Small) and ( $\tau i$ is Small)

If (Operation Point is High) then ( $k p$ is Big) and ( $\tau i$ is Medium Big)

If (Operation Point is Medium High) then ( $\mathrm{kp}$ is Medium Big) and ( $\tau$ i is Big)
If (Operation Point is Very High) then ( $k p$ is Very Big) and ( $\tau i$ is Very Big)

\section{RESULT AND DISCUSSION}

Control system have been designed was simulated using the help of SIMULINK in MATLAB software. Simulation was made to evaluate characteristics of closed-loop system transient responses by using PIDfuzzy supervision and PID-gain scheduling. 


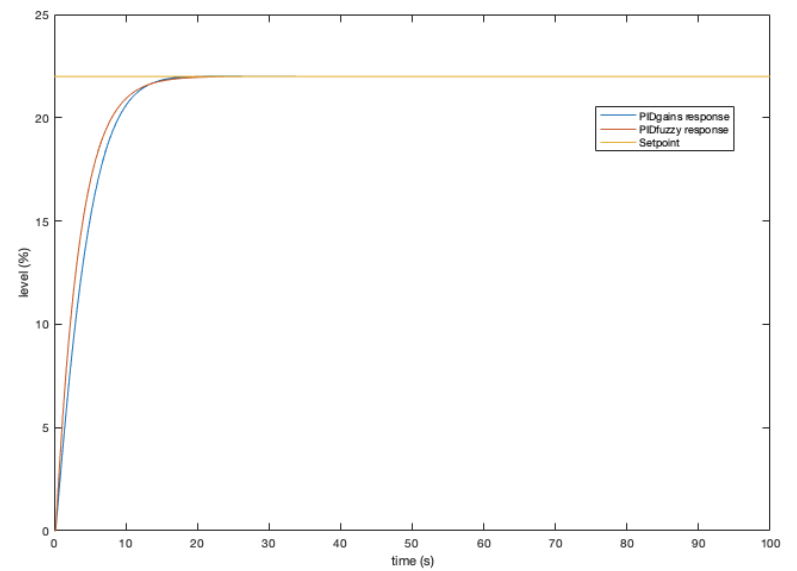

a

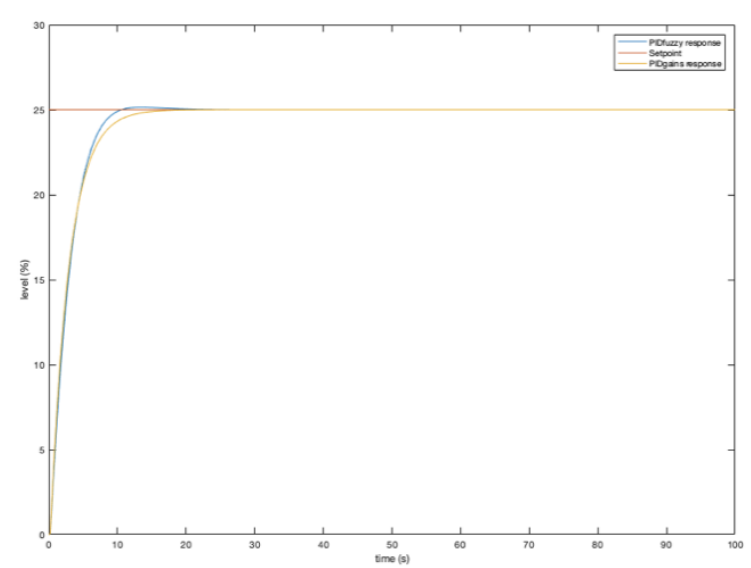

$\mathrm{b}$

Figure 2 PID-fuzzy supervision vs PID-gain scheduling responses with (a) set point value of 22 percent and (b) set point value of 25 percent

First, we give step signal value of 20 corresponding to the range of operation points (is 22 percent of level) consecutively to the set point. The control system responses can be seen at Figure 2(a). We calculated transient and steady-state response parameters as shown as Table 1. As we can see at the table, PID-fuzzy supervision has a better performance than PID-gain scheduling. PID-fuzzy supervision gave a faster response with 0.9 second of time constant deviation and 0.7 second of settling time deviation against PID-gain scheduling. In addition, we also calculate Integral Time-weighted Absolute Error (ITAE) of these two control system responses. The result is also confirmed that PID-fuzzy supervision is better. The ITAE of PID-fuzzy supervision $(249,4779)$ is sufficiently smaller than ITAE of PID-gain scheduling (307,0614).
Furthermore, we give another value of step signal, 25 (corresponding to $25 \%$ level) and get control system response as can see at Figure 2(b). The transient and steady-state response parameters is shown in Table 2. There are no significant changes in the result, still, PIDfuzzy supervision is better than PID-gain scheduling. But, in this point, there is a consideration about an overshoot in response. As we can see in Figure 4, there is an overshoot in the response of PID-fuzzy supervision with $0.5 \%$ of value of maximum overshoot (Mp). Certainly, this value is relatively small enough, but still, it's always depended on the system requirement. If the system has a boundary value of parameters which must not be passed (i.e., safety limitation etc.) then this Mp must be noticed, it is still within boundary or not.

Table 1. Response parameters with set point value of 22 percent and 25 percent

\begin{tabular}{lccccc} 
& \multicolumn{2}{c}{ Set Point Value of 22 Percent } & \multicolumn{2}{c}{ Set Point Value of 25 Percent } \\
Parameter & PID - Fuzzy & PID - & Gain & PID - Fuzzy & PID - Gain \\
Supervision & Scheduling \\
\hline Time constant $(\mathbf{s})$ & 3,6 & 4,5 & 3,1 & $\mathbf{3}$ \\
Delay time $(\mathbf{s})$ & 2,4 & 3,1 & 2,1 & $\mathbf{2}$ \\
Settling time $( \pm \mathbf{5} \%)(\mathbf{s})$ & 10 & 10,7 & 7,3 & $\mathbf{8 , 4}$ \\
Max. overshoot $(\%)$ & 0 & 0 & 0.5 & $\mathbf{0}$ \\
Error Steady-state $(\%)$ & $\mathbf{0}$ & $\mathbf{0}$ & $\mathbf{0}$ & $\mathbf{0}$ \\
\hline
\end{tabular}

By use the same statistical performance evaluation method, ITAE, we get 175,6932 of ITAE value for PIDfuzzy supervision and 199,4776 of ITAE for PID-gain scheduling. So, PID-fuzzy supervision is better because have a smaller ITAE.

The future prospect of this research is additional process disturbance consideration. Process disturbances must be modeled so it can be considered in controller design. Other future prospect is in the PID design. There are some methods that can be used for the design, like internal model control, 2nd order control etcetera. Some artificial intelligent (AI) methods like neural network, genetic algorithm etc. can be used for PID calculation to obtain more appropriate parameters. However, the use of AI must be done in offline in interest of a lower hardware requirements.

\section{CONCLUSION}

In our paper we investigate a level system as a nonlinear system. Two methods have been introduced: 
PID-gain scheduling and PID-fuzzy supervision. These control methods have been designed and simulated by using Simulink MATLAB to evaluate the characteristics of closed-loop system transient responses. The simulation result shown that the PID-fuzzy supervision has a better performance than the PID-gain scheduling. In operation point of 22 percent, PID-fuzzy supervision gives a faster response with 0.9 second of time constant deviation and 0.7 second of settling time deviation against PID-gain scheduling. In operation point of 25 percent, despite an overshoot must be noticed, PID-fuzzy supervision is still better than PID-gain scheduling.

\section{ACKNOWLEDGMENT}

This research was supported by Politeknik Energi dan Mineral (PEM) Akamigas. We thank our students and laboratory assistants who helped during the research.

\section{REFERENCES}

[1] J. F. Kanaga and P.S.H. Jose, Repetitive Controller for A Nonlinear Liquid Level System, IEEE $2^{\text {nd }}$ International Conferenceaon Innovations in Information, Embedded and Communication System, 2015.

[2] H. Kala, P. Aravind and M. Valluvan, Comparative Analysis of Different Controller for a Nonlinear Level Control Process, IEEE Conference on Information and Communication Technologies, 2013.

[3] C. Sanchez-Lopez et al. PID Controller Design Based On Memductor. International Journal of Electronics and Communication, 2019.

[4] J.G. Ziegler and N.B. Nichols and, OptimumaSettingsaforaAutomaticaControllers, Journal of Dyn. Sys., Meas. and Con., pp. 220-222, 1993.
[5] K. Astrom and T. Hagglund, PID Controller: Theory, Design and Tuning, 2nd ed, Library of Congress Cataloging-in-Publication Data, pp. 120$134,1994$.

[6] D. Chen and D.E. Seborg, PI/PID Controller Design Based on Direct Synthesis and Disturbance Rejection, Ind. Eng. Chem., vol. 41, PP. 4807-4822, 2002.

[7] Sudarshan K. Valluru and Madhusudan Singh, Performance Investigations of APSO Tuned Linear and Nonlinear PID Controllers for A Nonlinear Dynamical System, Journal of Electrical System and Information Technology, 2017.

[8] Y. Ren, Z. Li and F. Zhang, A New Nonlinear PID Controller and its Parameter Design, World Academy of Science, Engineering and Technology, pp. 882-887, 2010.

[9] D. Hryniuk, I. Suhorukova and I. Orobei, Nonlinear PID Controller and Methods of its Setting, IEEE Conference of Electrical, Electronic, and Information Sciences, 2017.

[10] Ma, Fengyin, An Improved Fuzzy PID Control Algorithm Applied in Liquid Mixing System, Proc. of IEEE Int. Conference on Information and Automation, 2014.

[11] Li, Qingchun, A New PID Fuzzy Controller (Fuzzy $P(I+D))$, IEEE Int. Conf. on Information Manag., Innovation Manag. and Industrial Engineering, 2011.

[12] Zhao Peimin, Zhang Huajun and Tong Xinchi, The Design of Gain Scheduling PID Controller of The USV Course Control System, Chinese Automation Congress, 2018.

[13] Zhao, Yan, Research on Application of Fuzzy PID Controller in Two-Container Water Tank System Control, IEEE Int. Conf. on Machine Vision and Human Machine Interface, 2010. 